\title{
The Optimal Dividend Problem in the Continuous- Time Compound Binomial Model Under the Reinsurance Control
}

\author{
Yan Guo ${ }^{1}$, Dingjie Fu ${ }^{2}$ \\ ${ }^{1,2}$ Hebei University of Technology, School of Science, Tianjin300401, China
}

\begin{abstract}
In this paper, we study the optimal dividend problem in the continuous-time compound binomial model under the reinsurance control. For the first time, the continuous-time compound binomial model is proposed by the GX Wang, Y Lin, B Zhang, and the related theory of the continuous-time compound binomial model in the bankruptcy probability has been relatively mature. On this basis, First we introduce the concept of dividend and reinsurance, then discuss the optimal dividend problem of the continuous-time compound binomial model. This paper is divided into five parts: the first part is preface; the second part modifies the continuous-time compound binomial model and introduce the definition of the restricted dividend. The third part discusses some properties of the value function. The forth part derives the HJB equation and the corresponding optimal dividend strategy, and proves that the solution which satisfy $\mathrm{HJB}$ is the value function. The fifth part is summary content.
\end{abstract}

Keywords: continuous-time compound binomial model restricted dividend under the reinsurance control HJB equation value function

\section{Introduction}

Collective risk theory as a part of insurance mathematics, usually through the establishment of an insurance company's random risk model for mathematical description of insurance business, and then use relevant mathematical theory to deal with risk issues. In general, in the risk model, we usually use a stochastic point process $\{N\}$ as the numbers of claims up to time $t$,a column of nonnegative random variables $\left\{U_{i}\right\}$ expressed the size of the $i$ th claim. Given an initial surplus $\mu$, the free surplus $X(t)$ of the insurance company at time $t$ can be written as

$$
X(t)=X(0)+Q(t)-S(t)
$$

Where $Q(t)$ expressed the premium income up to time $t$, $S(t)$ expressed the cumulative claim sizes up to time $t$ ,usually

$$
S(t)=\sum_{i=1}^{N_{t}} U_{i}
$$

The surplus progress in the continuous-time compound binomial modal can be written as

$$
X(t)=u+p t-\sum_{i=1}^{N_{t}} U_{i}
$$

And it satisfies the following conditions:

1) The free surplus $X(0)=u, u \in R^{+}$;

2) The premium rate $p$ is constant, and $Q(t)=p t$;

3) The claim sizes $\left\{U_{i}\right\}$ are identically distributed. Assume that they are the same distribution with $U$, and the distribution function can be written as $F_{U}$, the expection can be written as $\mu_{\mathrm{U}}$.

4 ) The counting process $N$ is continuous time binomial process with parameter $q$ : when the state reaches $k p \delta, k=1,2, \cdots$, the claim is paid by the insurance company with the probability $q \cdot \mathrm{n}_{t}=\left\lfloor\frac{t}{\delta}+\left(\frac{\mu}{c \delta}\right)\right\rfloor$ expressed the most amount of claims paid up to time $t$, so we can get

$$
\mathrm{P}\left(N_{t}=\mathrm{k}\right)=\mathrm{C}_{\mathrm{n}_{t}}^{k} q^{k}(1-q)^{n_{t}-k}, k=1,2, \cdots, n_{t} .
$$

5 ) The counting process $N_{t}$ and the stochastic variables $\left\{U_{i}\right\}$ are mutually independent;

6) It satisfies the conditions: $q \mu_{\mathrm{U}}<p \delta$;

7 ) $X(t)$ is an adapted right continuous with left limits stochastic process.

Correction of the continuous-time compound binomial model

In order to meet the requirements of this article, we modified the traditional continuous-time compound binomial model: when the state reaches $k \delta, k=1,2, \cdots$, the claim is paid by the insurance company with the probability $q \cdot \mathrm{n}_{t}=\left\lfloor\frac{t}{\delta}\right\rfloor$ expressed the most amount of claims paid up to time $t$, so we can get

$$
\mathrm{P}\left(N_{t}=\mathrm{k}\right)=\mathrm{C}_{n_{t}}^{k} q^{k}(1-q)^{n_{t}^{\prime}-k}, k=1,2, \cdots, n_{t} .
$$

We added time variable $t$, so the surplus process $\{(X(t), t)\}$ satisfies Markov property, the associated controlled surplus process is defined as $\{(x(t), t)\}$

$$
X(t)=x+p(t-s)-\sum_{i=N_{,+1}}^{N_{N}} U_{i}, t \geq s
$$

\section{Optimal Dividend Payments with}




\section{International Journal of Science and Research (IJSR) \\ ISSN (Online): 2319-7064}

Index Copernicus Value (2013): 6.14 | Impact Factor (2015): 6.391

We assume that the insurance company has the possibility of reinsurance. This means that the insurance company need a portion of the premium income paid to reinsurance company, When the insurance company have claim occurs, reinsurance company should undertake some of the claims.

Questions about reinsurance:

1)A Borel measurable function $R:[0, \infty) \rightarrow[0, \infty)$ that satisties $0 \leq R(\alpha) \leq \alpha$, where $R(\alpha)$ is the part of the claim paid by the insurance company when the size of the claim is $\alpha$, the reinsurance company covers $\alpha-R(\alpha)$.

2) The premium rate $q_{R}$ paid to the reinsurance company.So the premium rate left to the insurance company is $p_{R}=p-q_{R}$.

3)A control strategy is a process $\pi=\left(R_{t}, L_{t}\right)$, where $R_{t}$ is a reinsurance strategy, $L_{t}$ is the cumulative amount of dividends paid out from $\mathrm{s}$ to $\mathrm{t}$.

4) The control strategy is admissible, if it satisties the following conditions:

- The process $R_{t}$ is predictable; that is, the function $(\omega, \alpha) \rightarrow R_{t}(\omega, \alpha)$ is $f_{-} \times$Borel measurable for every $t \geq s$.

- The process $L_{t}$ is predictable, nondecreasing,

c a glad.

- For any $t \geq s$, the process $L_{t}$ verifies

$$
L_{t} \leq x+\int_{s}^{t} p_{R_{v}} d v-\sum_{i=N_{s}+1}^{N_{t}} R_{\tau_{i}}\left(U_{i}\right) .
$$

We denote by $\Pi$ the set of all the admissible control strategies. Given an admissible control strategy $\pi$, the controlled risk process $X^{\pi}(t)$ is given by

$$
X^{\pi}(t)=x+\int_{s}^{t} p_{R_{v}} d v-\sum_{i=N_{+}+1}^{N_{t}} R_{\tau_{i}}\left(U_{i}\right)-L_{t} .
$$

Where $\tau_{i}$ is the time of occurrence of the $i$ th claim.

We define the corresponding ruin time $\tau^{\pi}$ of the company as

$$
\tau^{\pi}=\inf \left\{t \geq 0: X^{\pi}(t)<0\right\}
$$

and the return function $V_{\pi}(x, t)$ as the cumulative expected discounted dividends from $s$ to $t$ with initial reserve $x \geq 0$. We can write $V_{\pi}(x, t)$ as

$$
V_{\pi}(x, s)=E_{(x, s)}\left(\int_{s}^{\tau^{\pi}} e^{-c(t-s)} d L_{t}\right),
$$

Where $c>0$ is the discount factor.

The optimal return function is defined as

$V(x, s)=\sup \left\{V_{\pi}(x, s), \pi \in \Pi\right\}, x \geq 0$

To simplify notation we define $V(x, s)=0, x<0$

\section{Basic Properties of the Value Functions}

Proposition 2.1 The optimal value function $V(x, s)$ is well defined and satisfies

$$
x+\frac{p}{c+\beta} \leq V(x, s) \leq x+\frac{p}{c} .
$$

Proof: For any admissible strategy $\pi=\left(R_{t}, L_{t}\right) \in \Pi, t \geq s$, we have

$$
\begin{aligned}
& L_{t} \leq x+\int_{s}^{t} p_{R_{v}} d v \leq x+\int_{s}^{t} p d v \leq(x+p(t-s)) I_{\{t \geq s\}} \\
& \varphi(t)=(x+p(t-s)) I_{\{t \geq s\}}
\end{aligned}
$$

Than, since $e^{-c t}$ is a positive and decreasing function,

$V_{\pi}(x, s) \leq E_{(x, s)}\left(\int_{s}^{\infty} e^{-c(t-s)} d \varphi(t)\right)=x+p \int_{s}^{\infty} e^{-c(t-s)} d t=x+\frac{p}{c}$

By definition

$$
V(x, s)=\sup \left\{V_{\pi}(x, s), \pi \in \Pi\right\} \leq x+\frac{p}{c} .
$$

So $V(x, s)$ is well defined and satisfies the second inequality of the proposition.

Let us prove now the first inequality. Given the initial condition $(x, s)$, consider the admissible strategy $\pi_{0}=\left(R_{0}, L_{0}\right) \in \Pi$, where $R_{0} \equiv R_{I}$, that is, does not take reinsurance strategy, $L_{0}$ which pays $x$ as a lump sum at time $s$ and than pays the incoming premium as dividends until the first claim, More precisely, $L_{0}=x+p(t-s)$. for $t \leq \tau_{1}$, Then we have

$V_{\pi_{0}}(x, s)=x+E_{(x, s)}\left(\int_{s}^{\tau_{1}} e^{-c(t-s)} d L_{t}\right)=x+\frac{p}{c+\beta}$.

So by definition we get the result.

Proposition 2.2 The optimal value function $V(x, s)$ satisfies

$y-x \leq V(y, s)-V(x, s) \leq V(x, s)\left(e^{c\left(t_{0}-s\right)}(1-q)^{-n_{s}}-1\right)$

For $y>x \geq 0$.

Proof. (1). Given $\varepsilon>0$, take an admissible stratefy $\pi=\left(R_{t}, L_{t}\right) \in \Pi_{(x, s)}^{R . L}, \quad$ such that $V_{\pi}(x, s) \geq V(x, s)-\varepsilon$. for $y>x \geq 0$, we define a new strategy

$\bar{\pi}=\left(R_{1}, L_{1}\right) \in \Pi_{(y, s)}^{R . L}$ : pay immediately $(y-x)$ as dividends at time $s$ and then follow the strategy $\pi$, The $\pi$ is admissible and we have

$V(y, s) \geq V_{-}(y, s)=V_{\pi}(x, s)+(y-x) \geq V(x, s)-\varepsilon+(y-x)$

So we obtain the first inequality

$V(y, s)-V(x, s) \geq y-x$

Let us prove the second inequality. Given initial stat $(y, s),(x, s)$, where $y>x \geq 0$ and $\varepsilon>0$, consider an admissible strat $\pi \in \Pi_{(y, s)}^{R . L}$ such that $V_{\pi}(y, t) \geq V(y, t)-\varepsilon$. Take now the strategy $\bar{\pi} \in \Pi_{(x, s)}^{R . L}$ which, starting with state $(x, s)$, pay no dividends and does not take reinsurance 


\section{International Journal of Science and Research (IJSR) \\ ISSN (Online): 2319-7064}

Index Copernicus Value (2013): 6.14 | Impact Factor (2015): 6.391

strategy if $X^{\bar{\pi}}(x, s)<y$ and follow strategy $\pi$ after current state $X^{\pi}(x, s)$ reaches $y$.The strategy $\pi$ is admissible. In the even of no claims, the surplus $X^{\pi}(x, s)$ reaches $y$ at time $t_{0}=s+\frac{y-x}{p}$; then, since the probability of reaching $y$ before the arrival of the first claim is $P\left(N_{t}=0\right)=C_{n}^{0} q^{0}(1-q)^{n_{s}}=(1-q)^{n_{s}}$.

we get

$V(x, s) e^{c\left(t_{0}-s\right)}(1-q)^{-n} \geq V\left(y, t_{0}\right) \geq V(y, s)$.

So

$V(y, s)-V(x, s) \leq V(x, s)\left(e^{c\left(t_{0}-s\right)}(1-q)^{-n_{s}}-1\right)$.

Where $n_{s}=\left\lfloor\frac{t_{0}-s}{\delta}\right\rfloor$.

Hence we obtain the result.

\section{HJB Equations and Verification}

Lemma 3.1 For any $(x, s)$ and any stopping time $\varsigma$, we can write

$V(x, s)=\sup _{\pi \in \Pi_{(,+,)}^{n+,}} E_{(x, s)}\left[\int_{s}^{\zeta \wedge \tau^{*}} e^{-c(t-s)} d L_{t}+e^{-c\left(\varsigma \wedge \tau^{*}-s\right)} V\left(X_{\tau^{*} \wedge \varsigma}^{\pi}, \varsigma \wedge \tau^{\pi}\right)\right]$

Proof. We proof this lemma for the case $\varsigma$ equal to a fixed time $T \geq s$. We call

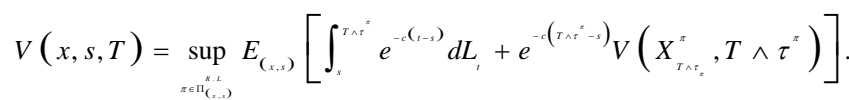

Let us prove first that $V(x, s) \leq V(x, s, T)$. Take any admissible strategy $\pi \in \Pi_{(x, s)}^{R . L}$, we can write

$$
\begin{aligned}
& V_{\pi}(x, s)=E_{(x, s)}\left[\int_{s}^{T \wedge \tau^{*}} e^{-c(t-s)} d L_{t}+e^{-c\left(T \wedge \tau^{*}-s\right)} V\left(X_{T \wedge \tau^{\pi}}^{\pi}, T \wedge \tau^{\pi}\right)\right] \\
& \leq E_{(x, s)}\left(\int_{s}^{T \wedge \tau^{*}} e^{-c(t-s)} d L_{t}\right)+E_{(x, s)}\left(e^{-c(T-s)} V\left(X_{T}^{\pi}, T\right) I_{\left\{\tau^{\tau}>T\right\}}\right) \\
& =V(x, s, T)
\end{aligned}
$$

By definition we get the result.

Proving by the same method, we can get

$$
V(x, s) \geq V(x, s, T)
$$

So we get the result.

Theorem 3.2: The optimal value function $V(x, t)$ satisfies the above properties and respectively about $x, t$ left and right differentiable left and right derivative satisfy HJB equations

$$
\left\{\begin{array}{l}
\sup _{t \in A}\left\{l(x, t)+\frac{\partial V(x, t)}{\partial t}+(c-l(x, t)) \frac{\partial V(x, t)}{\partial x}-\theta V(x, t)\right\}=0, t \neq k \delta \\
\Delta V(x, k \delta)+p \int_{0}^{x} V(x-y, k \delta) d F_{U}(y)-p V(x, k \delta)=0, t=k \delta
\end{array}\right.
$$

Where $\Delta V(x, k \delta)=V(x, k \delta)-V\left(x, k \delta_{-}\right)$.

Proof.1) Now we consider $t \neq k \delta$. By lemma 3.2.1 we have , for any stopping time $\zeta$, we get

$V(x, t) \geq E_{(x, t)}\left[\int_{t}^{\varsigma \wedge \tau} e^{-\theta(s-t)} l\left(X_{s}^{L}, s\right) d s\right]+e^{-\theta(\varsigma \wedge \tau-t)} V\left(X_{\varsigma^{\wedge} \tau}^{L}, \varsigma \wedge \tau\right) \mathrm{T}$ here exists $h$ such that $t<h<\tau$ and $[t, h] \in(k \delta,(k+1) \delta)$, so we get

That is

$$
\begin{aligned}
& 0 \geq E_{(x, t)}\left(\frac{\int_{t}^{h} e^{-\theta(s-t)} l\left(X_{s}^{L}, s\right) d s}{(h-t)}\right)+e^{-\theta(h-t)} \frac{\left(V\left(X_{h}^{L}, h\right)-V\left(X_{h}^{L}, t\right)\right)}{(h-t)} \\
& +e^{-\theta(h-t)} \frac{\left(V\left(X_{h}^{L}, t\right)-V(x, t)\right)}{(h-t)}+\frac{\left(e^{-\theta(h-t)}-1\right)}{(h-t)} V(x, t)
\end{aligned}
$$

Taking $h \rightarrow t$, we have

$$
0 \geq l(x, t)+\frac{\partial^{+} V(x, t)}{\partial t}+(c-l(x, t)) \frac{\partial^{+} V(x, t)}{\partial x}-\theta V(x, t)
$$

Since there exists a admissible strategy $l^{*}$ satisfies

$$
0=l^{*}(x, t)+\frac{\partial^{+} V(x, t)}{\partial t}+\left(c-l^{*}(x, t)\right) \frac{\partial^{+} V(x, t)}{\partial x}-\theta V(x, t)
$$

So the value function $V(x, t)$ satisfies

$$
\sup _{L \in \mathrm{A}}\left\{l(x, t)+\frac{\partial^{+} V(x, t)}{\partial t}+(c-l(x, t)) \frac{\partial^{+} V(x, t)}{\partial x}-\theta V(x, t)\right\}=0 .
$$

In the same way, we can get

$$
\sup _{L \in A}\left\{l(x, t)+\frac{\partial^{-} V(x, t)}{\partial t}+(c-l(x, t)) \frac{\partial^{-} V(x, t)}{\partial x}-\theta V(x, t)\right\}=0 \text {. }
$$

So we get the result.

2) Next we discuss $t=k \delta, k=1,2, \cdots$

By the definition of value function, we can get

$V\left(x, k \delta_{-}\right)=p \int_{0}^{\infty} V(x-y, k \delta) d F_{U}(y)+(1-p) V(x, k \delta)$.

Since $x<0$, we have $V(x, t)=0$, so

$V\left(x, k \delta_{-}\right)=p \int_{0}^{x} V(x-y, k \delta) d F_{U}(y)+(1-p) V(x, k \delta)$.

$V\left(x, k \delta_{-}\right)-V(x, k \delta)+p \int_{0}^{x} V(x-y, k \delta)-V(x, k \delta) d F_{U}(y)=0$.

By 1),2) we get the result.

Theorem 3.3 Assume that $\varphi(x, t)$ is increasing about $x$, piecewise continuous about $t$, has a jump at $t=k \delta$, and satisfies HJB equation. So $\varphi(x, t)=V(x, t)$ and the corresponding optimal strategy is $l^{*}$. 


\section{International Journal of Science and Research (IJSR) \\ ISSN (Online): 2319-7064}

Index Copernicus Value (2013): 6.14 | Impact Factor (2015): 6.391

Proof. Since the risk process $\left(\mathrm{X}^{L}(\mathrm{~s}), s\right)$ satisfies $\mathrm{X}_{t}^{L}=x$, so we have

$$
e^{-\theta(h-t)} \varphi\left(X_{h}^{L}, h\right)-\varphi\left(X_{t}^{L}, t\right)=e^{-\theta(h-t)} \varphi\left(X_{h}^{L}, h\right)-\varphi(x, t)
$$

Taking expectation and comparing with HJB equation, we obtain

$$
0 \geq E_{(x, t)}\left[e^{-\theta(h-t)} \varphi\left(X_{h}^{L}, h\right)-\varphi(x, t)+\int_{t}^{h} e^{-\theta(s-t)} l\left(X_{s}^{L}, s\right) d s .\right.
$$

Then

$$
\varphi(x, t) \geq E_{(x, t)} \int_{t}^{h} e^{-\theta(s-t)} l\left(X_{s}^{L}\right) d s+e^{-\theta(h-t)} \varphi\left(X_{h}^{L}, h\right)
$$

Taking $h \rightarrow \tau$ and combining with the boundary conditions $\varphi\left(X_{\tau}^{L}, \tau\right)=0$, we get

$\varphi(x, t) \geq E \int_{t}^{\tau} e^{-\theta(s-t)} l\left(X_{s}^{L}, s\right) d s=V^{L}(x, t)$.

When the dividend strategy $L$ is optimal strategy $L^{*}$, we get

$$
\varphi(x, t)=E \int_{t}^{\tau} e^{-\theta(s-t)} l^{*}\left(X_{s}^{L}, s\right) d s .
$$

So

$$
\varphi(x, t)=\sup _{L \in \mathrm{A}} V^{L}(x, t)=V(x, t) .
$$

Let us analysis of the optimal strategy $L^{*}$ :

By the HJB equation, we get

$$
\sup _{L \in A}\left\{\left(1-\frac{\partial V(x, t)}{\partial x}\right) l(x, t)+\frac{\partial V(x, t)}{\partial t}+\mathrm{c} \frac{\partial V(x, t)}{\partial x}-\theta V(x, t)\right\}=0 \text {. }
$$

Since $l\left(X_{s}^{L}, s\right) \quad$ satisfies $\quad 0 \leq l\left(X_{s}^{L}, s\right) \leq l_{0}$. when $1-\frac{\partial V(x, t)}{\partial x}<0$, the equation is decreasing about $l(x, t)$ , so we take $l^{*}(x, t)=0$; when $1-\frac{\partial V(x, t)}{\partial x}>0$, the equation is increasing about $l(x, t)$, so we take $l^{*}(x, t)=l_{0}$ , that is

$$
l^{*}(x, t)=\left\{\begin{array}{c}
0, \frac{\partial V(x, t)}{\partial x}>1, \\
\in\left[0, l_{0}\right], \frac{\partial V(x, t)}{\partial x}=1, \\
l_{0}, \frac{\partial V(x, t)}{\partial x}<1,
\end{array}\right.
$$

\section{References}

[1] Fang Y, Wu R. Optimal Dividend Strategy in the Compound Poisson Model with Constant Interest. Stochastic Models,2007,23:149-166.

[2] Jeanblanc-Picque M,Shiryaev A N. Optimization of the flow of dividends.Uspekhi Mat. Nauk 1995,50(2(302)):25-46.

[3] Gerber H,Shiu E.On optimal dividends:from reflection to refraction. J.Comput. Appl.Math.2006,186(1):4-22.

[4] Asmussen S,Taksar M.Controlled diffusion models for optimal dividend pay out.Insurance:Mathematics and
Economics, 1997,20:1-15.

[5] Dickson D C M, Waters H R. Some optimal dividends problems. ASTIN Bulletin.2004,34:49-74.

[6] Lin X S,Sendova K P.The compound Poisson risk model with multiple thresholds. Insurance Math. Econom.2008,42:617-627.

[7] Schmidli H. Stochastic Control in Insurance. Springer, New York,2008.

[8] Gerber H U, Shiu E S W, Smith N. Maximizing Dividends without Bankruptcy. Astin Bulletin,2006b,36(1):5-23.

[9] Li Y, Liu G X. Optimal dividend and capital injection strategy and optimal reinsurance strategy in the classical risk model. Mathematical Finance. 11-50.

[10] Hou Z T, Liu G X. Markov Skeleton processes and Their Applications. Science Press and International Press,2005.

[11]Ci Mei Sun, GX Liu. The Ruin Probability of Continus-time Compound Binomal Model Perturbed by Diffusion. 2007,20-35.

[12] Gerber H U,Shiu E S W On optimal dividend strategies in the compound Poisson model. Nam. Actuar. J.,2006,10(2):76-93.

[13] Kulenko N, Schmidli H. Optimal dividend strategies in a Cramer-Lundberg model with capital injections. Insurance: Mathematics and Economics, 2008,43(2):270-278.

[14] Albrecher H, Thonhauser S. Optimality results for dividend problems in insurance. Rev. R. Acad.Cien. Serie A. Mat.,2009,103(2):295-320.

[15] Shuaiqi Zhang, Guoxin Liu, Meici Sun. Ruin probabilities in the continuous-time compound binomial model with investment. Acta Mathematica Scientia $2015,35 \mathrm{~B}(2): 313-325$. 